\title{
Detection of third and sixth cranial nerve palsies with a novel method for eye tracking while watching a short film clip
}

\author{
Uzma Samadani, MD, PhD, ${ }^{1,2}$ Sameer Farooq, ${ }^{2}$ Robert Ritlop, MEng, ${ }^{2}$ Floyd Warren, MD, ${ }^{1,3}$ \\ Marleen Reyes, BA, ${ }^{1,2}$ Elizabeth Lamm, BA, ${ }^{2,4}$ Anastasia Alex, BS, ${ }^{3}$ Elena Nehrbass, BS, ${ }^{1,2}$ \\ Radek Kolecki, MS, ${ }^{2}$ Michael Jureller, BS, ${ }^{2}$ Julia Schneider, ${ }^{2}$ Agnes Chen, BA, ${ }^{2}$ Chen Shi, BS, ${ }^{1,2}$ \\ Neil Mendhiratta, BA, ${ }^{1,2}$ Jason H. Huang, MD, ${ }^{5}$ Meng Qian, PhD, ${ }^{6}$ Roy Kwak, MD, ${ }^{1}$ \\ Artem Mikheev, MS, ${ }^{7}$ Henry Rusinek, PhD, ${ }^{7}$ Ajax George, MD, ${ }^{7}$ Robert Fergus, PhD, ${ }^{4}$ \\ Douglas Kondziolka, MD, ${ }^{2}$ Paul P. Huang, MD, ${ }^{2}$ and R. Theodore Smith, MD, PhD ${ }^{3}$
}

${ }^{1}$ New York Harbor Health Care System, Manhattan Veteran's Administration; Departments of 2Neurosurgery, ${ }^{3}$ Ophthalmology, ${ }^{6}$ Psychiatry, and ${ }^{7}$ Radiology, New York University School of Medicine; ${ }^{4}$ Department of Computer Science, Courant Institute, New York University, New York, New York; and ${ }^{5}$ Department of Neurosurgery, Scott and White Health Care, Temple, Texas

\begin{abstract}
OBJECT Automated eye movement tracking may provide clues to nervous system function at many levels. Spatial calibration of the eye tracking device requires the subject to have relatively intact ocular motility that implies function of cranial nerves (CNs) III (oculomotor), IV (trochlear), and VI (abducent) and their associated nuclei, along with the multiple regions of the brain imparting cognition and volition. The authors have developed a technique for eye tracking that uses temporal rather than spatial calibration, enabling detection of impaired ability to move the pupil relative to normal (neurologically healthy) control volunteers. This work was performed to demonstrate that this technique may detect CN palsies related to brain compression and to provide insight into how the technique may be of value for evaluating neuropathological conditions associated with CN palsy, such as hydrocephalus or acute mass effect.
\end{abstract}

METHODS The authors recorded subjects' eye movements by using an Eyelink 1000 eye tracker sampling at $500 \mathrm{~Hz}$ over 200 seconds while the subject viewed a music video playing inside an aperture on a computer monitor. The aperture moved in a rectangular pattern over a fixed time period. This technique was used to assess ocular motility in 157 neurologically healthy control subjects and 12 patients with either clinical CN III or VI palsy confirmed by neuro-ophthalmological examination, or surgically treatable pathological conditions potentially impacting these nerves. The authors compared the ratio of vertical to horizontal eye movement (height/width defined as aspect ratio) in normal and test subjects.

RESULTS In 157 normal controls, the aspect ratio (height/width) for the left eye had a mean value \pm SD of $1.0117 \pm$ 0.0706 . For the right eye, the aspect ratio had a mean of $1.0077 \pm 0.0679$ in these 157 subjects. There was no difference between sexes or ages. A patient with known CN VI palsy had a significantly increased aspect ratio (1.39), whereas 2 patients with known CN III palsy had significantly decreased ratios of 0.19 and 0.06 , respectively. Three patients with surgically treatable pathological conditions impacting $\mathrm{CN} \mathrm{VI}$, such as infratentorial mass effect or hydrocephalus, had significantly increased ratios $(1.84,1.44$, and 1.34, respectively) relative to normal controls, and 6 patients with supratentorial mass effect had significantly decreased ratios $(0.27,0.53,0.62,0.45,0.49$, and 0.41 , respectively). These alterations in eye tracking all reverted to normal ranges after surgical treatment of underlying pathological conditions in these 9 neurosurgical cases.

CONCLUSIONS This proof of concept series of cases suggests that the use of eye tracking to detect CN palsy while the patient watches television or its equivalent represents a new capacity for this technology. It may provide a new tool for the assessment of multiple CNS functions that can potentially be useful in the assessment of awake patients with elevated intracranial pressure from hydrocephalus or trauma.

http://thejns.org/doi/abs/10.3171/2014.10.JNS14762

KEY WORDS abducent nerve; diplopia; eye movement tracking; hydrocephalus; oculomotor nerve; prepontine cistern; diagnostic and operative techniques

ABBREVIATIONS CN = cranial nerve; EDH = epidural hematoma; ICP = intracranial pressure; pds = prism diopters; $\mathrm{SDH}=$ subdural hematoma; $\mathrm{TBI}=$ traumatic brain injury. SUBMITTED April 4, 2014. ACCEPTED October 27, 2014.

INCLUDE WHEN CITING Published online December 12, 2014; DOI: 10.3171/2014.10.JNS14762.

DISCLOSURE Dr. Samadani has intellectual property and equity in Oculogica, Inc., and Mr. Ritlop has equity in Oculogica, Inc. The aforementioned company did not contribute any funding to this study and had no influence over its design or this paper's content. 
$\mathrm{T}$ He cranial nerves (CNs) are known to be susceptible to changes in intracranial pressure (ICP) due to mass effect or hydrocephalus. Early detection of subclinical $\mathrm{CN}$ palsy in awake patients thus might prove useful for assessment of patients with hydrocephalus, possible shunt malfunction, or traumatic brain injury (TBI). We developed a novel algorithm for eye tracking performed while subjects watch a music video playing inside an aperture on a computer monitor. The aperture moves around the monitor periphery at a known rate so that the position of the pupil can be analyzed at any given time based on the time elapsed since the start of the video. By using elapsed time analysis, our method should be able to detect impaired ability to move the pupil relative to normal controls. Our algorithm sets the amount of time that the eye should be moving in any given direction essentially constant and then quantitates the distance that it travels. Because the method does not entail spatial calibration, it does not compensate for impaired motility and can be used in patients who do not follow commands, such as those with aphasia, patients in a persistent vegetative state, and small children.

The CN III is classically impacted by supratentorial mass effect, which may lead to transtentorial herniation. ${ }^{1}$ A clinical indicator of transtentorial herniation is palsy of the oculomotor nerve due to compression by the uncus as the nerve courses along the tentorial notch after its exit from the pontomesencephalic junction and prior to its entry into the cavernous sinus. Among other functions, the oculomotor nerve innervates the superior and inferior recti muscles of the orbit, rotating the eyeball up and down. A CN III palsy in the context of trauma or stroke and evidence of radiographic transtentorial herniation may potentially be an indication for neurosurgical intervention. ${ }^{29}$

The abducent nerve exits the ventral pontomedullary junction, courses through the prepontine cistern, and travels through the Dorello canal into the cavernous sinus before innervating the lateral rectus muscle of the orbit, lateralizing the pupil. ${ }^{26}$ It is known to be susceptible to myriad disorders, including ischemia, diabetes mellitus, trauma, and hydrocephalus. ${ }^{19}$

We hypothesized that our eye-tracking algorithm would detect clinical and subclinical palsies of the abducent and oculomotor nerves. To test this hypothesis we tracked eye movements in 157 normal control subjects to establish a range for normal eye movement ratios with our algorithm. We then compared the ratio of vertical versus horizontal eye movement for normal subjects to subjects with known pathological conditions of these nerves who were recruited from the ophthalmology and neurosurgery clinics. Patients with supratentorial lesions resulting in uncal mass effect who are assessed with this novel eye-tracking algorithm would be expected to demonstrate decreased vertical/horizontal eye movement relative to normal controls. Patients with infratentorial lesions or other pathological entities impacting CN VI would be expected to have increased vertical/horizontal eye movement ratios. We performed prospective measurement of that ratio in patients with confirmed CN III and VI palsies (positive controls) and in stable, awake neurosurgical patients undergoing procedures for supratentorial and infratentorial mass lesions.

\section{Methods \\ Subject Selection}

Subjects were recruited in accordance with institutional review board policy. Written informed consent was obtained from all subjects prior to participation.

Control subjects were employees, volunteers, visitors, and patients at the investigating institutions. Inclusion criteria for normal control subjects were as follows: age 7-100 years; vision correctable to within 20/50 bilaterally; intact ocular motility; and ability to provide a complete ophthalmological, medical, and neurological history as well as medications/drugs/alcohol consumed within the 24 hours prior to tracking. Parents were asked to corroborate details of the above for children 7-17 years old. Exclusion criteria were history of strabismus, diplopia, palsy of CN III, IV, or VI, papilledema, optic neuropathy, dementia or cognitive impairment, hydrocephalus, sarcoidosis, myasthenia gravis, multiple sclerosis or other demyelinating disease, active or acute epilepsy, stroke/hemorrhage, or brain injury sufficiently significant to result in hospitalization. Control subjects reporting any brain injury within the week prior to recruitment for eye-tracking tests were also excluded.

Patients were recruited for participation specifically because they were known to have palsy of CN III or VI, or mass lesions or pathological entities impacting CN III or VI. Mass lesion inclusion criteria were as follows: lesion apparent on radiographic imaging, clinical necessity for resection, and patient able to consent to and engage in eye tracking. Exclusion criteria were the same as for normal subjects, plus bilateral compression of optic nerves resulting in decreased visual acuity, visual field deficit, sellar lesion, and cavernous sinus invasion. Subjects who underwent neurosurgery were tracked pre- and postoperatively at their convenience, as described in the case descriptions. Ophthalmological examinations of neurosurgical patients were performed as described in the individual case descriptions. All of the surgically treated patients were examined by a board-certified, experienced attending neurosurgeon familiar with standard techniques for neurological examination.

Subjects who wore glasses were tracked without refraction correction, whereas those who wore contact lenses were tracked with the lenses in place. Subjects with toric contact lenses were asked to remove lenses for the tracking procedure. The eyes of each of the test subjects were tracked serially and separately; however, the visual stimulus was presented binocularly.

\section{Visual Stimulus}

Subjects' eye movements were recorded with an Eyelink 1000 eye tracker (SR Research) at a distance of 55 $\mathrm{cm}$ from a computer monitor over a fixed time period of 220 seconds. The visual stimuli were music videos including Shakira's "Waka-Waka" (World Cup soccer tribute); K'Naan's “Wavin' Flag (When I Grow Older I will be Stronger);" Michael Jackson's "Man in the Mirror;" "Hakuna Matata" (from the Lion King); and Shankar Ehsan Loy's "Bhumbroo." The videos played continuously in a square aperture with an area $1 / 8$ the screen size of 
the monitor. The aperture remained stationary at the upper left corner of the monitor screen for the first 10 seconds, then moved clockwise along the outer edges of a 17-inchdiameter monitor for 5 complete cycles of 40 seconds each plus an extra 10 seconds at the end of the last cycle. The first 10 and last 10 seconds of the full data set were discarded to yield 200 seconds of data. Sound volume was adjusted to the subject's preference.

\section{Data Analysis}

The eye tracker sampled pupil position at $500 \mathrm{~Hz}$, yielding 100,000 samples over 200 seconds. We created scatterplots of the entire time series by plotting the 100,000 (x, y) pairs representing the 2 orthogonal components of the instantaneous angle of pupil reflection over time to create "box trajectories" that reflected the cartesian location of the pupil over time. In control subjects, these figures look like boxes, reflecting the location of the aperture as it moved around the screen over time (Fig. 1, left column), with each cycle shown in a different color (red, green, cyan, magenta, blue).

\section{Aspect Ratio}

Elapsed time quantitation enables localization of the video aperture during measurement of eye positions. The panels on the left side of Fig. 1 demonstrate pupil position as the video traverses all 4 sides of the rectangular viewing monitor, with each cycle of the video shown in a different color (red, green, cyan, magenta, blue). The panels on the right side show only the mean coordinates of 5 cycles as the video traverses the top (red) or bottom (cyan) of the rectangular monitor. In each particular case (e.g., normal subject, CN VI palsy, epidural hematoma [EDH]), as the video traverses the top of the monitor over 10 seconds, the patients' pupils have a median value where the red dots are. As the video traverses the bottom of the monitor, the pupils have a median value where the cyan dots are. Thus, the height of the box is calculated as the median y cartesian coordinate during the first 10 seconds minus the median y coordinate during the third 10 seconds. The box height $(\mathrm{h})$ was defined as the difference $y 2-y 1$, where $y 2$ is the median of y cartesian coordinate values as the video traversed the top and $y 1$ is the median of y cartesian coordinate values as the video traversed the bottom of the monitor (Fig. 1 , left column). The box width (w) was similarly defined as $\mathrm{x} 2-\mathrm{x} 1$, with $\mathrm{x} 2=$ median $\mathrm{x}$ coordinate as the aperture traversed the right side, and $\mathrm{x} 1=$ the median $\mathrm{x}$ for the left side. The aspect ratio was defined as $\mathrm{h} / \mathrm{w}$. Thus, eye-tracking recordings for a person with decreased vertical relative to horizontal eye movement would yield a decreased aspect ratio. We defined an outlier as 2 SDs away from the mean, which was calculated using the mean function on Excel.

\section{Statistics Comparing Age and Sex Among Control Subjects}

A linear regression between aspect ratio and age was calculated and a regression t-test was used to determine whether the slope of the regression line was statistically significantly different from 0 . A Mann-Whitney-Wilcoxon test was used to determine if the true difference between the distribution of male aspect ratio and female aspect ratio was statistically significantly different from 0 .

\section{Perimesencephalic Cistern Volume}

The actual space around CN III as it travels from the interpeduncular cistern at the pontomesencephalic junction to the cavernous sinus is difficult to quantify with great accuracy. To overcome this limitation, we assessed preoperative CT scans for reduction in perimesencephalic cistern volume relative to the maximal-volume postoperative scan by using established segmentation methodology $^{36}$ originally adapted from MRI analysis. ${ }^{25}$ To correct for variable angulation of the skull on axial CT images that could be introduced by misalignment of the head between scans, we realigned and resampled each CT volume to a standard (template) coordinate system. This was done by marking in 3D space the left eye lens (LE), the right eye lens (RE), and the junction of the superior colliculi (C). In the template space these 3 corresponding structures $-\mathrm{LE}^{\prime}$ $\mathrm{RE}^{\prime} \mathrm{C}^{\prime}$-were positioned symmetrically such that the principal axis of the brainstem was in the $\mathrm{z}$ direction (across plane). We then computed the rigid body (volume-preserving) transformation ( $\mathrm{T}$ ) that best mapped the triangle $<\mathrm{LE}$ $\mathrm{RE} \mathrm{C}>$ onto the triangle $\left\langle\mathrm{LE}^{\prime} \mathrm{RE}^{\prime} \mathrm{C}^{\prime}>\right.$. The transformation $\mathrm{T}$ was used to resample the $\mathrm{CT}$ volume to the template space. The cistern was then segmented by placing a $4-\mathrm{cm}-$ diameter cylinder centered on the center of the brainstem and extending vertically (head-foot direction) from pineal gland to tuberculum sellae. For each voxel within the cylinder we computed the voxel's partial volume (Pv) of CSF according to its attenuation (A) measured in Hounsfield units (HU): $P v=\left(A_{\text {brain }}-A\right) /\left(A_{\text {brain }}-A_{\text {water }}\right)$. Here $A_{\text {brain }}$ $=48 \mathrm{HU}$, and $\mathrm{A}_{\text {water }}=0 \mathrm{HU}$. The Pv was constrained to [0-1]. The cistern volume was computed as the sum of $\mathrm{Pv}$ values within the cylinder multiplied by voxel volume, excluding the suprasellar cistern.

\section{Radiology Analysis}

Two experienced board-certified radiologists who were blinded to the eye-tracking findings evaluated the preoperative $\mathrm{CT}$ images to assess mass effect on CN III.

\section{Results}

The 157 control subjects were $52 \%$ male and had a mean age of $36.73 \pm 16.90$ years (mean \pm SD) and a range of 7-87 years. The mean aspect ratio for the left eye was $1.0117 \pm 0.0706$. For the right eye it was $1.0077 \pm 0.0679$. A sample result in a normal patient is shown in Fig. 1A (left). Below it, Fig. 1B is from a patient with diabetes who had a CN VI palsy demonstrating an aspect ratio of 1.39 ( $p<0.001$ ), showing that the height of the box is greater than its width. The bottom row, Fig. 1C, demonstrates calculation of aspect ratio in an awake patient with an acute $\mathrm{EDH}$. In this particular case the aspect ratio is actually negative, because over all 5 cycles as the video traverses the top of the rectangle the pupils are where the red dots are clustered, whereas as it traverses the bottom they are where the cyan dots are clustered.

Just as a patient with a CN VI palsy due to diabetes had an increased aspect ratio (Fig. 1B), 2 patients with CN III 
A
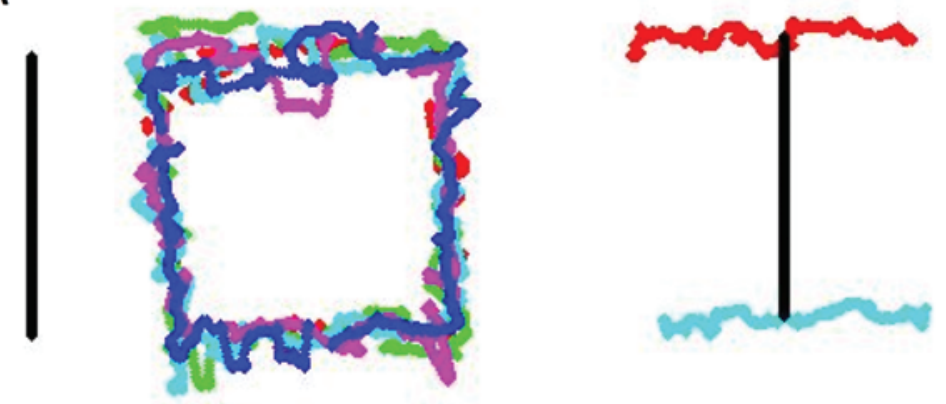

B
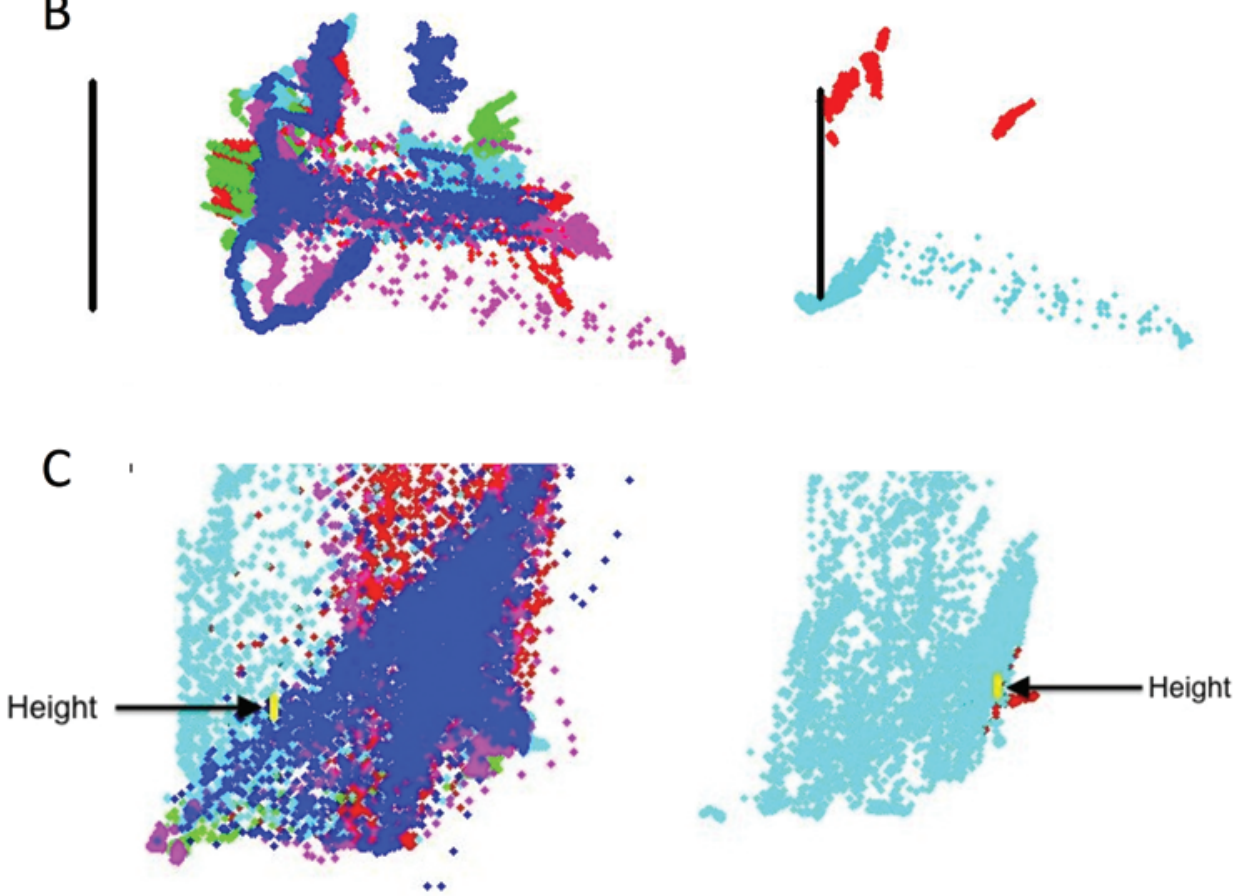

FIG. 1. Tracking eye movements to detect ocular dysmotility. The scatterplots show the eye position of 1 eye as the video travels around the computer monitor. On the left, the first cycle is plotted in red, the next in green, then cyan, magenta, and blue. A normal control eye movement trajectory follows the video aperture as it moves in a box shape. Aspect ratio is defined as height/width. The right side of the figure depicts how the height of the box is measured by obtaining the median values over 5 cycles as the video traverses the monitor during the 1 st and 3 rd 10 seconds of each cycle (plotted in red and cyan, respectively). The width would be obtained by calculating median values over 5 cycles during the 2 nd and 4 th 10 seconds of each cycle. The aspect ratio of an idealized control subject by definition should be 1.0 since the time spent traversing the top and bottom of the boxes (providing height) is equal to the time spent traversing the sides of the boxes (providing width). The mean aspect ratio for the eye tracked in panel A was 1.01. Panel B represents a diabetic patient with a CN VI palsy. This 56-year-old man with hemoglobin A1C values ranging from $8.7 \%$ to $9.4 \%$ (reference range $4.6 \%-6.5 \%$ ) presented with horizontal diplopia worse at distance and improved by covering or closing 1 eye. Visual acuity was $20 / 30$ and he had full motility on examination. He had esotropia of 20 prism diopters (pds), with 30 pds on left gaze and 12 pds on right gaze, consistent with a mild right CN VI palsy. Right eye tracking revealed an abnormal trajectory relative to a normal control with an aspect ratio equal to $1.39(p<0.001)$, suggesting decreased lateral pupil movement as the video traversed the top and bottom of the screen. Panel $\mathbf{C}$ depicts the box height analysis of a patient for whom the aspect ratio is negative, due to $\mathrm{CN}$ III compression from an acute EDH. Figure is available in color online only.

palsies, due to either tumor involvement or diabetes, had decreased aspect ratios (Fig. 2 left and right, respectively). These resulted in aspect ratios of 0.1870 (Fig. 2 left) and 0.05637 (Fig. 2 right), demonstrating decreased vertical relative to horizontal pupil movement.

In normal control subjects, regression analysis of aspect ratio by age revealed no association. Sex also did not impact aspect ratio on the Mann-Whitney-Wilcoxon test.

\section{Illustrative CN III Cases}

\section{Chronic Subdural Hematoma}

The patient was an 86-year-old right-handed man with a medical history of hypertension, hyperlipidemia, and mild chronic renal insufficiency, and an ophthalmological history of bilateral cataract surgery (2 years and 8 years prior), pseudophakia, and scleral buckling. He had a base- 



FIG. 2. Left: Eye tracking of a subject whose right CN III was surgically severed prior to its entrance into the cavernous sinus during resection of a tumor involving the nerve. A 76-year-old man with a medical history of glaucoma and myopia, and current presbyopia, had right upper eyelid ptosis and a pupil that was fixed and nonreactive to light or accommodation. He had a large-angle (50-pd) right exotropia and 14-pd right hypotropia in primary gaze. He was not able to adduct to the midline in the right eye, nor was there any significant vertical duction capacity in the right eye. Steristrips were applied to elevate the right upper lid and the patient underwent eye tracking which revealed an aspect ratio (height/width of box trajectory) equal to 1.0206 in the left eye and 0.1870 in the right eye ( $p<0.001$ for right eye). Right: Eye tracking of a subject with a right $\mathrm{CN}$ III palsy secondary to diabetes mellitus. A 79-year-old man with a history of hypertension and diabetes mellitus with hemoglobin $\mathrm{A} 1 \mathrm{C}$ values ranging from $8.2 \%$ to $11.4 \%$ over the past year (normal range $4.6 \%-6.5 \%$ ) presented to the emergency room complaining of 3 days of painful double vision. On examination he demonstrated right eyelid ptosis. His motility was intact in the left eye. In the right eye he had exotropia of $8 \mathrm{pds}$. The patient was then seen in follow-up 11 days later in clinic. At that time his examination remained unchanged except for decreased motility of the right eye at -4 adduction, -4 elevation, and -4 depression. Steristrips were applied to elevate the right upper lid and the patient underwent eye tracking, which revealed an aspect ratio (height/width of box trajectory) equal to 0.05637 in the right eye, suggesting that the right eye is an outlier from normal. Figure is available in color online only.

line visual acuity of 20/25 (right eye) and 20/30 (left eye). The patient took $81 \mathrm{mg}$ of aspirin per day prophylactically. The patient fell without losing consciousness and presented several weeks later with headache, but was otherwise neurologically well. A head CT was performed and demonstrated a small left-sided subdural hematoma (SDH) (Fig. $3 \mathrm{~A})$. Aspirin was discontinued and platelets were transfused. Eye tracking was performed and demonstrated 2 of 5 cycles with reduced vertical amplitude. The headache resolved spontaneously and the patient elected to undergo observation of this lesion. Eight days later the headache became exacerbated and a repeat CT scan demonstrated enlargement of the hematoma (Fig. 3B). The patient remained intact on neurological examination, exhibiting neither neglect nor pronator drift. His pupils remained symmetrical. Eye tracking was again performed and demonstrated decreased vertical amplitude on 5 of 5 cycles, with an aspect ratio of 0.27 . The patient underwent SDH drainage, during which $100 \mathrm{ml}$ of fluid was extracted using a closed twistdrill drainage system. Head CT and eye tracking was repeated after removal of the twist drainage apparatus (Fig. 3C). Despite persistence of residual SDH, the eye tracking vertical amplitude was normal. His headaches resolved and he became asymptomatic. The patient was discharged home after participating in physical therapy.
Perimesencephalic cistern compression (on immediate preoperative CT scan): right cistern $80 \%$ of baseline, left cistern $61 \%$ of baseline.

Radiologist 1: "The brainstem is shifted right, there is medialization of the left uncus, and it may barely touch cisternal CN III. Separately there is mass effect on the left cavernous sinus by the uncus (loss of CSF cleft)."

Radiologist 2: "The patient has a full uncus with medial displacement. There is mass effect on the cistern, but no overt compression of CN III."

\section{Acute EDH}

The patient was a 62-year-old, right-handed man with a medical history of goiter/iatrogenic hypothyroidism, hypertension, and hyperlipidemia, and an ophthalmological history of glaucoma controlled with latonoprost drops. He had 20/20 vision bilaterally. He presented with syncopal fall and loss of consciousness persisting a few minutes. At presentation he was awake, alert, and had fluent speech. His pupils were equal and extraocular movements remained intact. His head CT showed a small right frontoparietal EDH underlying a stellate skull fracture. Eye movement tracking was performed (Fig. 4). Five hours later his examination findings deteriorated and he developed drowsiness, dysarthria, left-sided neglect, and a left pronator drift. His pupils remained equal and extraocular movements remained intact by examination. He underwent craniotomy, evacuation of $\mathrm{EDH}$, and cranial plating of the skull fracture. Postoperatively his left-sided neglect and pronator drift resolved over the subsequent 48 hours. He scored 13/50 on the Executive Interview 25 Assessment Test (EXIT) on postoperative Day 7. Eye tracking was repeated on postoperative Days 7, 11, and 35. He was discharged home after 1 week of inpatient rehabilitation and returned to work 2 months after surgery.

Perimesencephalic cistern compression (on preoperative CT scan): right cistern $27 \%$ of baseline, left cistern $35 \%$ of baseline.

Radiologist 1: "There is contact with CN III on the right side."

Radiologist 2: "There is no evidence of overt CN III compression."

\section{Right Frontal Metastasis}

A 63-year-old man, a 100-pack-year smoker who had declined to see a physician for his entire adult life and thus reported no relevant medical or ophthalmological history, presented to the emergency room with a cough, requesting treatment for a flulike illness. On examination he was noted to be disoriented and to have a mild left hemiparesis. Pupils were equal and reactive, and extraocular movements appeared intact. Head CT demonstrated a right frontal mass, and chest radiograph showed a large left upper lobe chest mass. The patient was administered Decadron (10 mg orally every 6 hours), and a CT scan of the chest/abdomen/pelvis (not shown) and brain MRI studies (Fig. 5A) were obtained. Eye movement tracking was performed 48 hours after admission, immediately before right frontal craniotomy for resection of a moderately well-differentiated squamous cell carcinoma metastasis. 

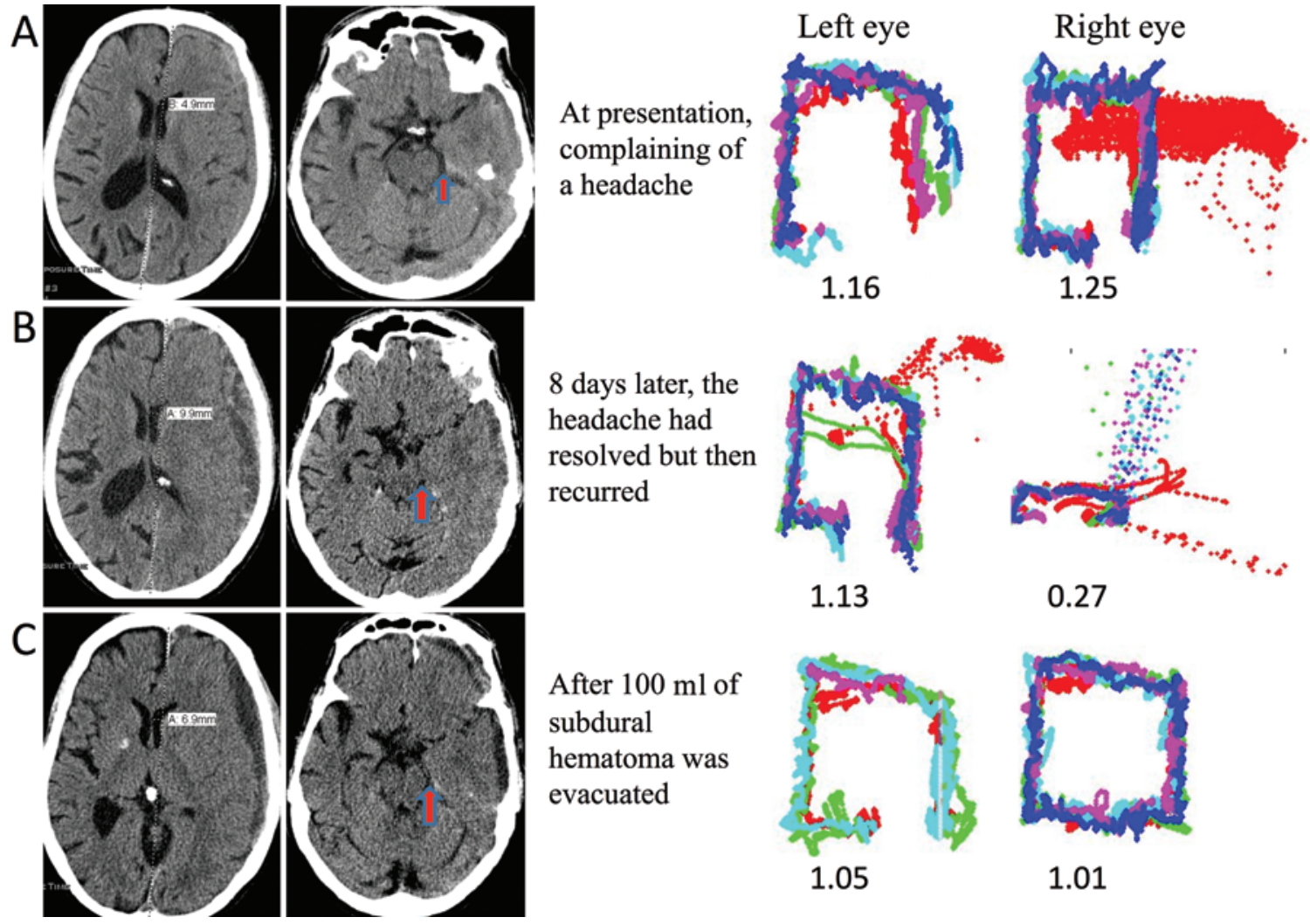

8 days later, the headache had resolved but then recurred

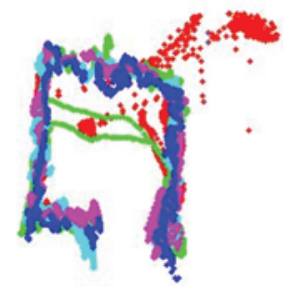

1.13

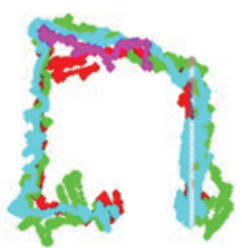

1.05

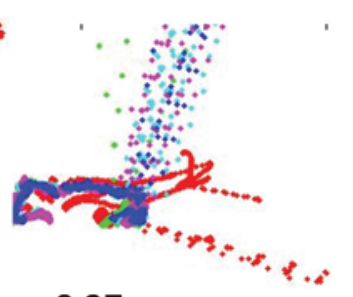

0.27

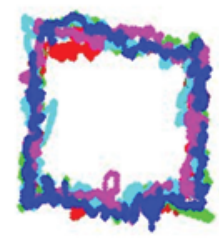

1.01

FIG. 3. Exacerbation and reversibility of pupillary vertical amplitude in a patient with SDH. An 86-year-old man with a history of cataracts presented with headache several weeks after a fall. Initial axial CT scans showed a small SDH (A, left) with an open perimesencephalic cistern (arrow). Eight days later his headache became exacerbated, and repeat imaging demonstrated that the SDH had expanded, creating mass effect on the brain and pushing the medial aspect of the temporal lobe into the perimesencephalic cistern (B, arrow). A twist-drill drain was placed and $100 \mathrm{ml}$ of subdural fluid was evacuated, resulting in resolution of the mass effect on the perimesencephalic cistern (C, arrow). Figure is available in color online only.

A radiographic gross-total resection was performed. The patient went home 1 week after his craniotomy and underwent repeat eye tracking at a follow-up visit 1 month later. He subsequently underwent radiation therapy to his whole brain. He declined treatment for the lung mass and died 7 months after his initial diagnosis.

Perimesencephalic cistern compression (on immediate preoperative CT scan): right cistern $77 \%$ of baseline, left cistern $73 \%$ of baseline.

Radiologist 1: "There is subtle shift of the uncus towards the midline. It likely does not contact cisternal CN III, but may press on the ipsilateral cavernous sinus, since the CSF cleft adjacent to the sinus is gone."

Radiologist 2: "The uncus is medially deviated; there is no overt compression on CN III."

\section{Right SDH}

The patient was an 87-year-old man with a medical history of hypertension, hyperlipidemia, diabetes, chronic renal insufficiency, prostate cancer treated with radiation, and cerebrovascular disease treated with left carotid endartectomy. His ophthalmic history was significant for pseudophakia, glaucoma, left eye cataract, and allergic conjunctivitis. His visual acuity was $20 / 20$ and $20 / 25$. He took aspirin $(81 \mathrm{mg})$ sporadically. The patient presented with left leg weakness resulting in a fall. On examination he had mild left hemiparesis without neglect. Platelets were transfused, eye tracking was performed, and a twistdrill drainage procedure was performed. More than 200 $\mathrm{ml}$ subdural fluid was removed. The patient's left hemiparesis resolved and he was discharged to rehabilitation. Sixteen days after the procedure, repeat eye tracking was performed.

Perimesencephalic cistern compression (on immediate preoperative CT scan [Fig. 5B]): right cistern $61 \%$ of baseline, left cistern $73 \%$ of baseline.

Radiologist 1: "Cisterns are effaced but there is no direct mass effect on CN III."

Radiologist 2: "There is mass effect, but no overt compression of CN III."

\section{Left Glioblastoma Multiforme}

The patient was a 67-year-old man with a medical history of prostate cancer, hypertension, hyperlipidemia, alcoholism in remission, and gunshot wound to the left shoulder with retained missile fragment. His ophthalmic baseline was 20/40 vision in the right eye and 20/50 in the left eye. He presented with 2 months of stuttering and right arm and hand weakness. He had a witnessed seizure on the day of presentation that began with shaking 




Preoperative CT images
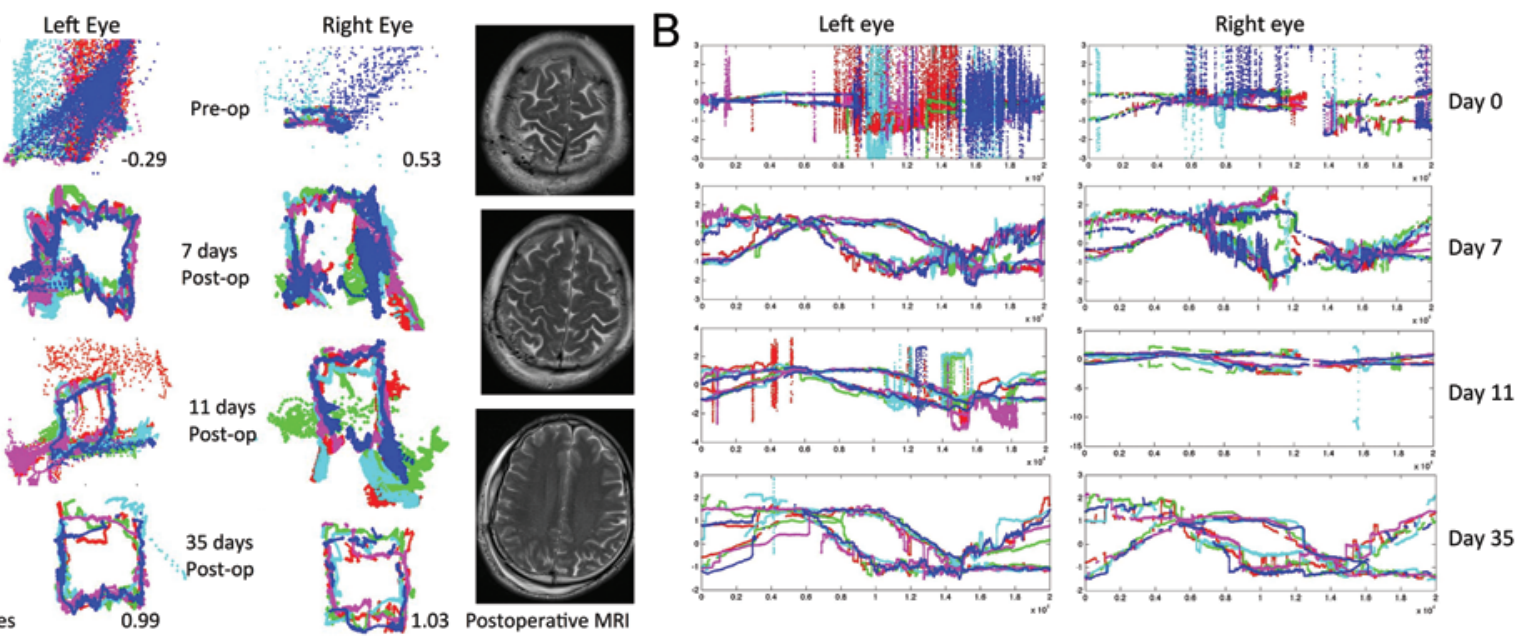

FIG. 4. A 62-year-old patient with an acute EDH and bilateral perimesencephalic compression after a fall. The patient presented with a neurologically nonfocal examination and CT scans showing a small right frontoparietal EDH and nondisplaced skull fracture. On presentation he was verbally appropriate without focal neurological deficit. Pupil size was equal by gross examination and extraocular movements appeared intact when eye tracking was performed. Four hours later the patient developed a left pronator drift and intermittent dysarthria. We performed EDH evacuation and fracture repair. He underwent repeat eye tracking on postoperative Days 7, 11, and 35. Aspect ratio on the day of presentation was negative for the left eye. Ratios are listed on the figure for the day of presentation and postoperative Day 35. Conventional scatterplots are shown in panel A. Panel B depicts the eye tracking as a plot of the cartesian coordinate of the pupil on the $y$ axis and time on the $x$ axis, with each 40-second cycle in a different color. Such a plot reveals that the position of the pupil is altered at roughly the same points during each cycle as the video circumnavigates the monitor 5 times. This suggests that the patient is awake during tracking because the pupils are not lost and that the alterations are not coincidental but driven by a common intrinsic pathological entity, because they repeatedly occur at the same place in 5 consecutive cycles. Figure is available in color online only.

of the right upper extremity and progressed to generalized tonic-clonic activity. On examination he had intact pupils and extraocular movements. His speech was slow with paraphasic errors and difficulty with repetition and naming. Head CT scans obtained with contrast revealed a left frontotemporal cystic mass (Fig. 5C). The patient underwent eye tracking and subsequently underwent awake stereotactic drainage of the cyst, which revealed necrotic cells and was nondiagnostic for malignancy, followed by awake stereotactic craniotomy with speech mapping for resection of a glioblastoma multiforme. Gross-total resection was achieved radiographically. The patient had preserved speech but mild hemiparesis postoperatively and participated in rehabilitation prior to discharge home. He underwent repeat eye tracking at 1 month postoperatively. He received Temodar and radiation therapies as an outpatient and remained independent in activities of daily living, with no tumor recurrence at 7 months postoperatively.

Perimesencephalic cistern compression (on immediate preoperative CT scan): right cistern $42 \%$ of baseline, left cistern $39 \%$ of baseline.

Radiologist 1: "Cisterns patent. No uncal shift. No contact with CN III."

Radiologist 2: "No evidence of CN III compression."

\section{Intracranial Abscess}

A 65 -year-old man with a medical history of hypertension, hyperlipidemia, coronary artery disease, and posttraumatic stress disorder, with no known ophthalmic disorders and visual acuity of 20/20 bilaterally presented 2 weeks after left parietal craniotomy for an esophageal junction metastasis was performed by a surgeon at another institution, with worsening right hand coordination and ataxia. On examination the patient had right pronator drift and hemineglect. A CT scan revealed edema at the surgical site and MRI studies (Fig. 5D) revealed a peripherally enhancing collection in the previous tumor cavity. Eye tracking was performed and then the patient was taken to the operating room for reexploration craniotomy and evacuation of an abscess deep to the dura mater. He was treated with antibiotics for 12 weeks postoperatively. Eye tracking was then repeated.

Perimesencephalic cistern compression (on immediate preoperative CT scan): right cistern $79 \%$ of baseline, left cistern $84 \%$ of baseline.

Radiologist 1: "Cisterns patent. No uncal shift. No contact with CN III."

Radiologist 2: "No evidence of CN III compression."

None of the patients with supratentorial lesions who were found to have restricted vertical amplitude on eye tracking reported diplopia or other symptoms of disconjugate gaze. All of the patients were reported to have normal ocular motility on examination by a neurosurgeon or ophthalmologist, which suggests that the palsies are subclinical.

\section{Illustrative CN VI Cases \\ Calvarial Mass With Cerebellar Compression}

A 54-year-old man presented with a medical history of poorly differentiated papillary thyroid carcinoma resected 5 and 3 years prior and then treated with radioactive iodine and whole-beam radiation therapy. He reported a tender 

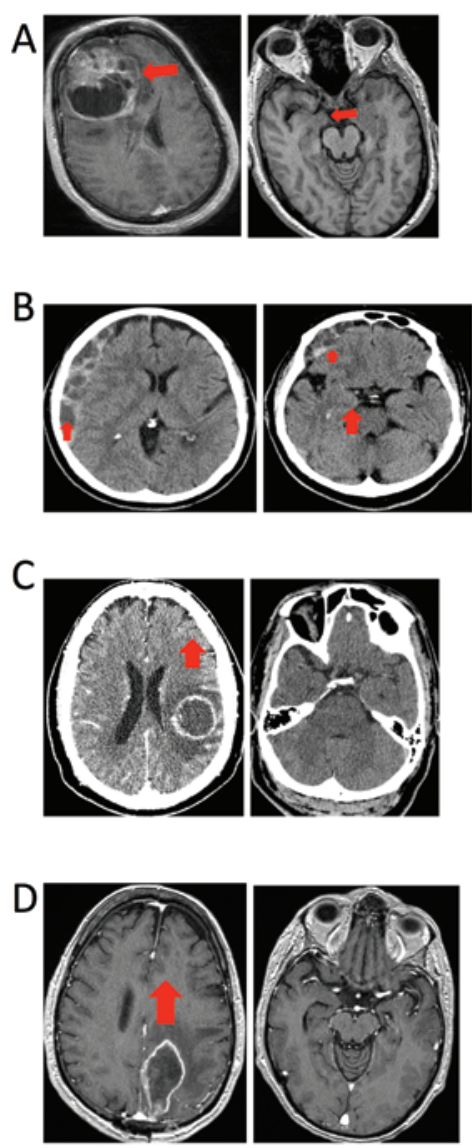

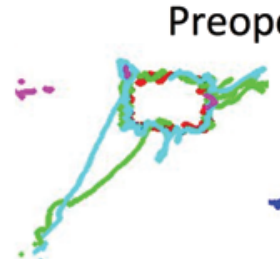

.62

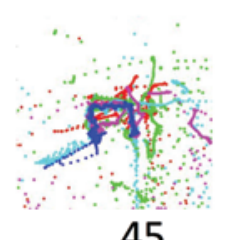

.45

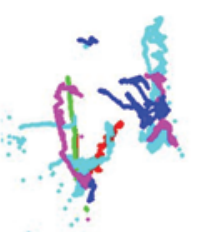

.76



1.06
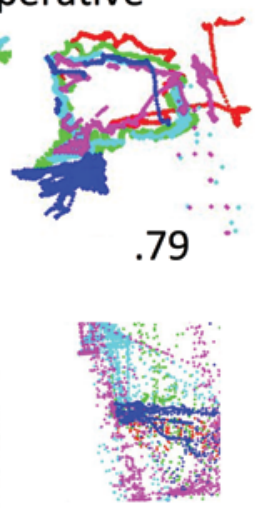

.45
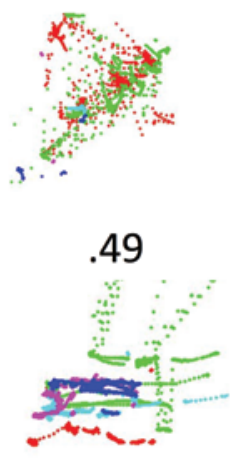

.41
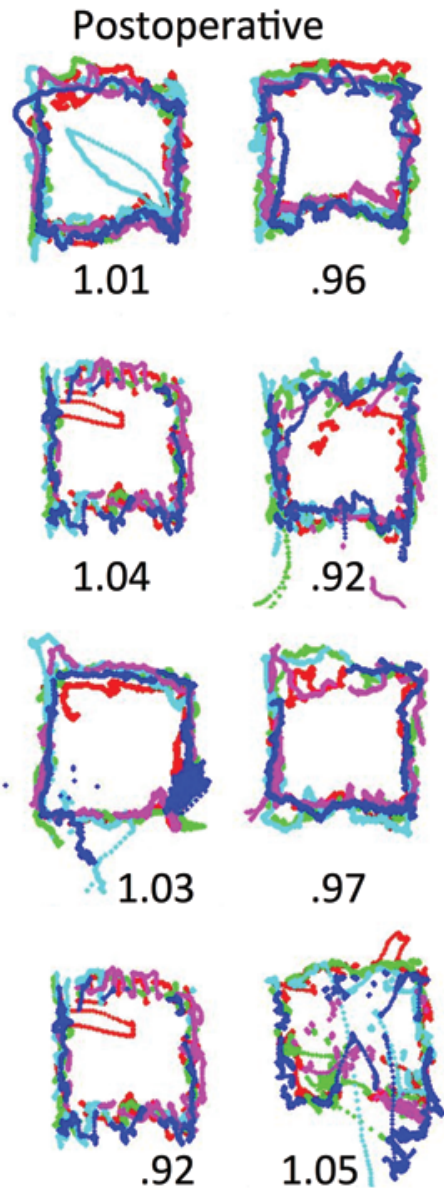

Left eye

Right eye

Left eye

Right eye

FIG. 5. Preoperative imaging and pre- and postoperative eye tracking of 4 subjects with supratentorial mass lesions. Aspect ratios are provided within the figure. A: Axial MRI sequences obtained in a 63-year-old man with a right frontal brain mass. B: Head CT scans obtained in an 87-year-old man with an SDH. C: Head CT scans obtained in a 67-year-old man with a glioblastoma multiforme. D: Axial MRI studies obtained in a 65-year-old man with an intracranial abscess. The single arrows designate the lesions in all images (and the second, large arrow in B right indicates uncal mass effect). Figure is available in color online only.

mass on the back of his head and a progressive occipital headache that was improved by supine positioning. The patient denied nausea, vomiting, dizziness, diplopia, or vision difficulty. Physical examination by a neuro-ophthalmologist demonstrated normal extraocular motility and no evidence of papilledema. Dilated funduscopic examination revealed sharp, pink, and flat optic discs with a cup/ disc ratio of 0.3 in both eyes. Imaging revealed a calvarialbased mass (Fig. 6A and B). There was narrowing of the right prepontine cistern on MRI (Fig. 6C, arrow), but no transependymal flow to suggest hydrocephalus (Fig. 6D). Eye tracking was performed (Fig. 6, right panel, upper row). His aspect ratio of 1.84 in the left eye was highly significantly greater than normal controls. The patient underwent craniectomy for resection of the mass, and cranioplasty reconstruction. Eye tracking was repeated on postoperative Day 1 (Fig. 6, right panel, lower row) and demonstrated return to the normal range in both eyes. $\mathrm{He}$ did well and was discharged home, neurologically well, on postoperative Day 3. This patient's preoperative eye tracking demonstrated an increased aspect ratio in the eye contralateral to the peripontine cistern narrowing on MRI scan that resolved after lesion resection.

\section{A Cerebellar Mass}

A 56-year-old man with a medical history significant for 35 years of cigarette smoking, daily marijuana use, and hypertension presented with dull occipital headaches that improved with ibuprofen, as well as blurred vision and an unsteady gait. Detailed ophthalmological examination revealed 20/25 visual acuity, normal ocular motility, and no evidence of papilledema, with sharp, pink, and flat optic discs and a cup/disc ratio 0.35 in both eyes. Chest radiograph revealed a mass in the right upper lobe. Brain imaging revealed a right cerebellar mass pushing the brainstem to the left and resulting in narrowing of the left prepontine cistern (Fig. 7A, arrow). There was no evidence of transependymal flow on T2-weighted MRI (Fig. 7B). Eye tracking was performed (Fig. 7, right panel, upper row) and revealed a highly significantly elevated aspect ratio of 1.44 in the right eye. Retrosigmoid suboccipital craniotomy was performed for resection of the brain tumor. Eye tracking was 

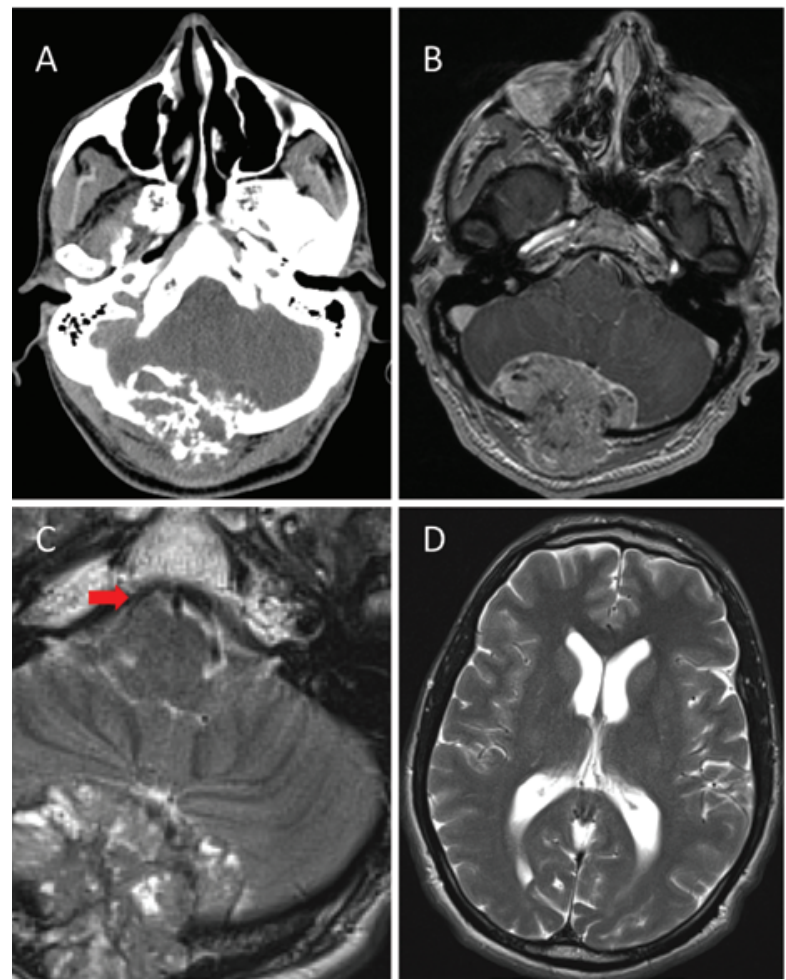

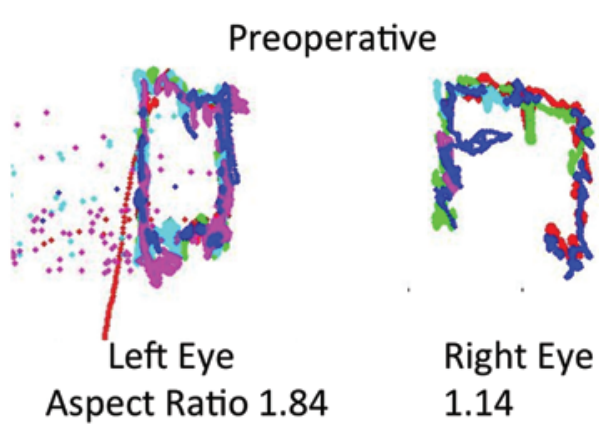

\section{Postoperative Day 1}

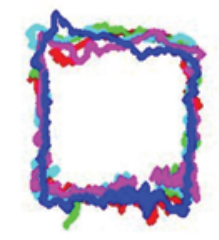

Left Eye

Aspect Ratio 1.03

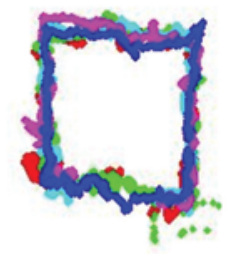

Right Eye

1.13

FIG. 6. Resection of a calvarial mass causing right prepontine cistern narrowing normalizes the aspect ratio. The imaging (A-D) demonstrates a large calvarial mass compressing the cerebellum and causing asymmetrical mass effect on the prepontine cistern. (The arrow in $\mathrm{C}$ indicates mass effect on CN VI.) Preoperative eye tracking is shown in the right panel, upper row, and demonstrates a highly significantly increased aspect ratio in the left eye as well as mildly abnormal tracking in the right eye. Eye tracking obtained on postoperative Day 1 is shown in the right panel, lower row, and demonstrates the return to normal aspect ratio in both eyes. Figure is available in color online only.

repeated on postoperative Day 1 (Fig. 7, right panel, lower row) and was normal in both eyes. The patient remained neurologically well and was discharged home on postoperative Day 4. In this patient, eye tracking demonstrated an increased aspect ratio in the eye contralateral to the peripontine cistern narrowing on the MRI study that resolved after lesion resection.

\section{Neurosarcoidosis}

A 59-year-old woman presented with a 1-year history of progressive intermittent vertigo, biparietal headache, and imbalance. She reported intermittent horizontal diplopia. Ophthalmological examination revealed full ocular motility, and no evidence of papilledema or neurosarcoidosis. Admission MRI of the brain revealed hydrocephalus without evidence of a discrete mass (Fig. 8 left). Eye tracking was performed preoperatively (Fig. 8, right panel, upper row) revealing a highly significantly decreased aspect ratio in the left eye (0.81) and a highly significantly increased aspect ratio in the right eye (1.34) relative to normal controls. A ventriculoperitoneal shunt was placed, relieving the headaches. Eye tracking was repeated on postoperative Day 2 (Fig. 8, right panel, lower row) and revealed return to the range of normal in both eyes. The angiotensin-converting enzyme I level in CSF was elevated, at 4.8 $\mathrm{U} / \mathrm{L}$ (reference range 0-2.5 U/L). Subsequent evaluation revealed increased gallium uptake on lung scan and mediastinal lymphadenopathy on chest CT. The patient was scheduled to undergo biopsy of her lung mass for evaluation of sarcoidosis.

\section{Discussion}

Many methodologies exist for assessment of CNS function, ranging from physical examination to radiographic imaging and electrical activity assessment. Nonetheless, some pathological conditions, such as elevated ICP, concussion, and shunt malfunction remain difficult to diagnose, even with invasive means.

We present the eye-tracking results in 157 subjects without known significant neurological or ophthalmological pathological conditions. These subjects watched a video that traveled the perimeter of a rectangular viewing monitor at a speed of 10 seconds per edge. The test was very easily tolerated and no subjects had to be excluded due to inattention. In an idealized model, the ratio of vertical to horizontal eye movement would be equal to 1.00 because the time spent traveling vertically versus horizontally should be the same. We noted that normal subjects had mean ratios of 1.01 for both the left and right eyes, with a relatively low standard deviation of approximately $7 \%$. There was no significant difference between the eyes in these controls.

We then demonstrated that subjects with CN III palsies or supratentorial mass effect had decreased vertical/ horizontal eye movement ratios and that subjects with $\mathrm{CN}$ VI palsies, infratentorial mass effect, or hydrocephalus 

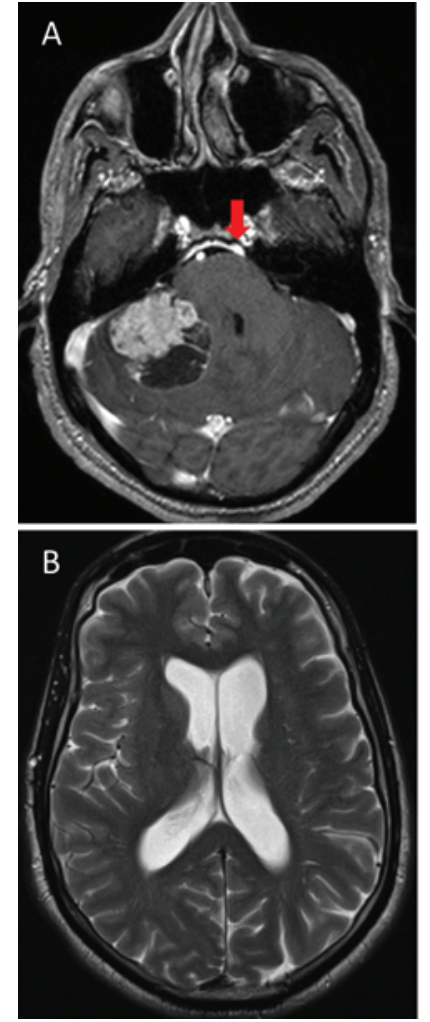

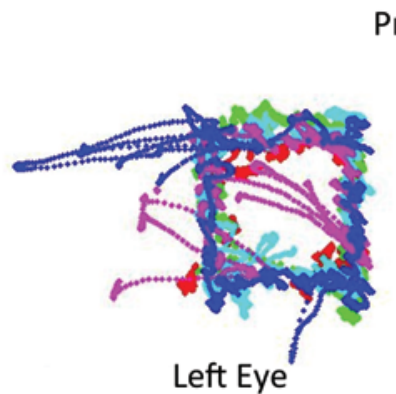

Aspect Ratio 1.03

\section{Preoperative}

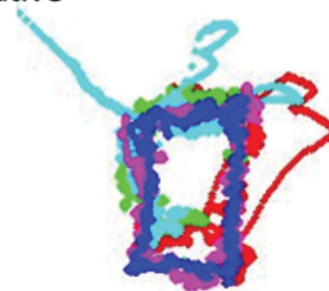

Right Eye

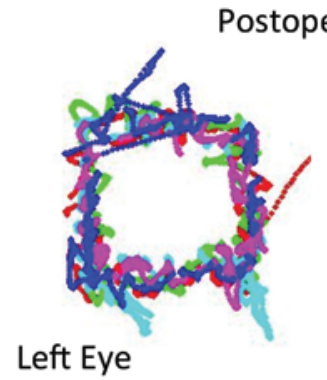

Aspect Ratio 0.97

FIG. 7. Resection of a right cerebellar mass causing left prepontine cistern narrowing normalizes the aspect ratio. The imaging (A and B) demonstrates a large cerebellar mass compressing the left prepontine cistern, which is designated by an arrow. Preoperative eye tracking is shown in the right panel, upper row, and demonstrates a highly significantly increased aspect ratio in the right eye. Eye tracking obtained on postoperative Day 1 is shown in the right panel, lower row, and demonstrates the return to normal aspect ratio. Figure is available in color online only.

had increased ratios, consistent with the activities of the affected nerves.

\section{Cranial Nerve III}

Our data suggest that we have developed an eye-tracking algorithm that captures the decreased vertical ocular motility associated with CN III palsy while a subject watches a brief video of their choice that moves within an aperture on a viewing monitor (Figs. 2 and 3). Patients with supratentorial mass lesions demonstrated low outlying value aspect ratios preoperatively, which reverted to normal postoperatively (Figs. 4 and 5). Because a low aspect ratio suggests decreased vertical relative to horizontal movement of the eye, such a finding may be mediated via CN III. Our algorithm was sensitive enough to detect supratentorial mass effect confirmed by an algorithm quantitating perimesencephalic cistern volume, regardless of whether uncal mass effect was noted by a radiologist.

Our algorithm may potentially be clinically useful for the early detection of supratentorial mass effect and transtentorial herniation. Determining the exact mechanism underlying the phenomenon we are observing will be the subject of future studies. In every patient with a supratentorial pathological entity in whom we performed eye tracking, the abnormal findings occurred while tracking of the eye contralateral to the entity. This has occurred consistently in our entire database of patients and regardless of whether the afferent stimulus to the eyes is presented monocularly or binocularly. It is possible that CN III itself is the locus, perhaps by ischemia from pressure exerted in the interpeduncular fossa. When the lesion is only affecting 1 eye, as in many of the cases, it is less likely to be supranuclear. ${ }^{16,17,28}$ To impact only 1 eye, a subtentorial supranuclear lesion would have to invoke skew deviation, which does not arise in the course of midbrain compression from the mass effect of supratentorial lesions. ${ }^{7}$ Monocular paresis of ocular elevation can occur in patients with lesions near the posterior commissure, but this is very rare and does not supervene in cases of midbrain compression from the mass effect of supratentorial lesions. ${ }^{2,14,28}$

Perhaps the case best illustrating a patient with both supranuclear and probable CN III deficit is depicted in Fig. $5 \mathrm{C}$. This patient had a large left cystic mass and was moderately aphasic at presentation due to postictal state and Todd paralysis. The tracking in both eyes was abnormal but the aspect ratio was lower on the side contralateral to the lesion, as is seen in the other patients with supratentorial mass effect. If the lesion were purely supranuclear one might expect the aspect ratios to be relatively equal. Unequal ratios suggest an infranuclear mechanism.

Figure 5A and B depicts patients who clearly have more substantive mass effect than the others, and in both cases decreased vertical eye movement seems to be almost matched by decreased horizontal eye movement. These patients may also have decreased horizontal eye movement, reflecting CN VI palsy from either increased ICP or 


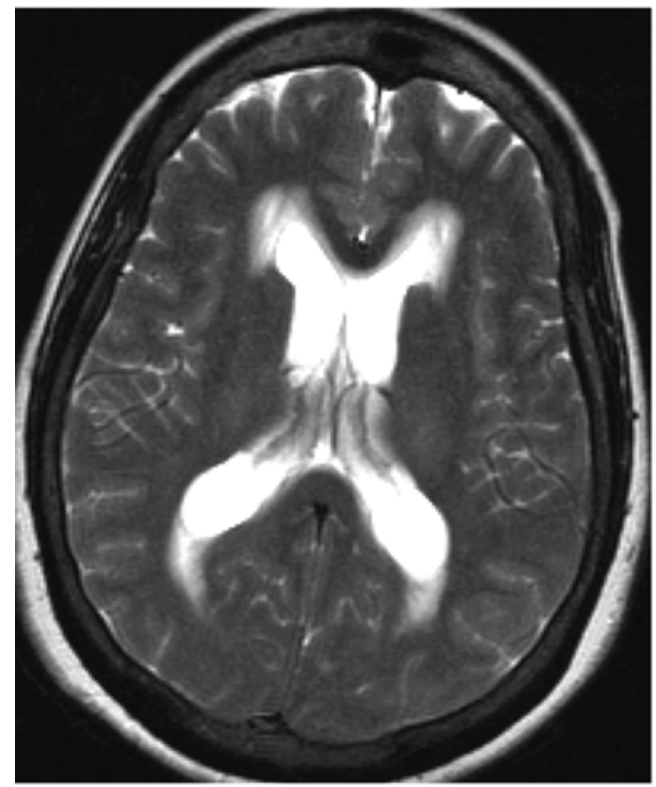



Left Eye

Aspect Ratio 0.81

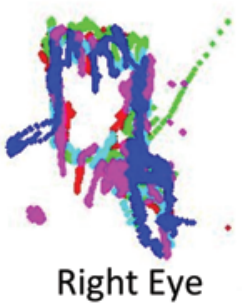

1.34

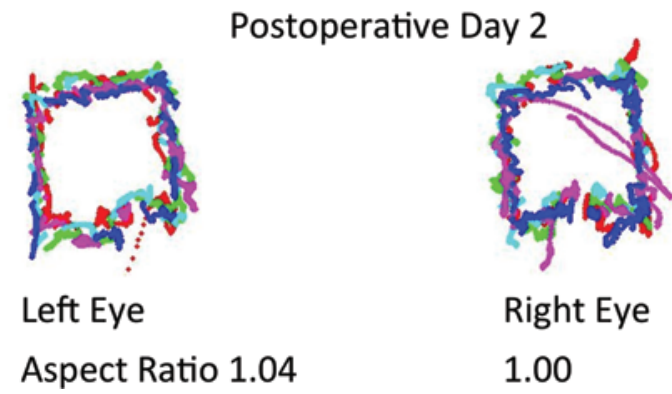

FIG. 8. Shunting of CSF in a patient with hydrocephalus presumably caused by sarcoidosis normalizes the aspect ratio. The MR image (left) was obtained in a patient with neurosarcoidosis resulting in hydrocephalus. Her eye tracking is abnormal in both eyes preoperatively (right, upper row), with an increased aspect ratio. This ratio returns to normal postoperatively (right, lower row). Figure is available in color online only.

direct mass effect on the nerve. Additionally, because both eyes are affected, there may be a supranuclear component to the altered tracking.

The patient with the greatest quantitative difference in perimesencephalic volume between his pre- and postoperative CT scan had bilateral decreases in pupillary vertical amplitude on eye tracking. This patient presented after a fall resulting in a right frontoparietal skull fracture and small EDH causing almost no subfalcine herniation (Fig. $4 \mathrm{~A}$, upper CT image) but reduction in perimesencephalic cistern volume to $27 \%$ of the postoperative value on the right side and $35 \%$ of postoperative volume on the left (lower CT image). Postoperatively, CT and MRI studies (Fig. 4A, right column) revealed no evidence of residual structural injury; however, the patient's eye box trajectory persisted in having increased variability, raising the possibility that the eye tracking is reflecting the impact of radiographically silent brain injury. The impairment of his cognitive capabilities after surgery is consistent with the association between mild brain injury and eye-tracking variability. Impaired reading ability in patients sustaining supratentorial brain injury or stroke has been attributed in part to deficits in fixation, saccade, and pursuit. ${ }^{10}$ Smooth pursuit during target blanking has been hypothesized to reflect shearing of cerebellar-cortical tracts, resulting from mild brain injury. ${ }^{33}$ Diffusion tensor imaging demonstrating disruption of white matter tracts also correlates with impaired visuomotor task completion. ${ }^{8}$

A potential limitation of the study could be that conditions other than supratentorial mass lesions can cause changes in vertical ocular motility. Myasthenia gravis, ${ }^{35}$ supranuclear palsies, ${ }^{5,6,11}$ and primary eye muscle patho- logical conditions such as congenital progressive external ophthalmoplegia ${ }^{22,31}$ are also going to produce abnormalities in vertical movements. In addition, disorders causing a restrictive problem impacting the orbit (thyroid orbitopathy, 13,34 previous trauma, extraocular muscle infiltration, and inflammation) will also cause problems with eye movements that may involve more of the vertical (most frequently in the case of trauma) than the horizontal movements. None of the patients in our study had any history of these conditions, and in all of them their eye tracking improved to normal after surgery was performed to address their supratentorial mass lesions, suggesting that the lesion's mass effect may indeed be the cause of their motility dysfunction.

\section{Cranial Nerve VI}

We present a series of cases in which patients had increased vertical relative to horizontal movement of the eye when we used the same algorithm. Two patients had clinically apparent CN VI deficits confirmed by the neuro-ophthalmologist's examination. The other two did not have CN VI palsies on neuro-ophthalmological examination, and only 1 of these 2 had intermittent complaints of diplopia. The 2 with cerebellar mass effect had radiographic compression of CN VI. The patient with neurosarcoidosis had radiographic evidence of hydrocephalus, which can lead to stretching of CN VI. Because CN VI innervates the lateral rectus, and we note decreased horizontal relative to vertical movement in these patients relative to normal control subjects and similar to the first 2 subjects with confirmed $\mathrm{CN}$ VI palsy, our results are consistent with detection of subclinical CN VI palsy in these patients. The abducent 
nerve moves the pupil laterally, and these subjects demonstrated decreased lateral movement on eye tracking. In the 3 patients in whom the pathology could be surgically treated, these trajectories resembled normal controls after treatment.

One potential utility of this technology is detection of symptoms caused by hydrocephalus. Here we described 1 patient with hydrocephalus due to sarcoidosis. Her eye tracking was abnormal in both eyes preoperatively and returned to normal postoperatively. However, given the diverse baseline ocular pathological conditions in hydrocephalic patients, $, 3,15,37$ it is likely that tracking results will need to be compared with each patient's own normative data to be useful in that particular context. A second potential utility is the monitoring of awake patients with suspected elevated ICP, because CN VI is known to be susceptible to elevated ICP. ${ }^{19}$

In the 2 patients with tumors causing mass effect with compression of CN VI as it exits the pontomedullary junction through the prepontine cistern, the abnormal eye-tracking pattern must be due at least in part to either nuclear or nerve compression. If it were due to a supranuclear mechanism, it would most likely affect both eyes the same way. ${ }^{16}$ In the third illustrative CN III case, which was diagnosed in a 63-year-old man with right frontal metastasis, the patient had a highly significantly increased aspect ratio in the left eye and only a marginally increased one in the right eye. His calvarial lesion was sufficiently large that it may have been compressing both nerves, in unequal quantities. In the fourth illustrative CN III case, which was diagnosed in an 87-year-old man with right $\mathrm{SDH}$, the patient had only 1 eye with an increased aspect ratio preoperatively that returned to normal postoperatively. That these patients both improved by postoperative Day 1 after tumor resection suggests that their pathological condition was altered by the surgeries to remove these tumors.

\section{Implications and Limitations}

Ocular motility is commonly assessed in 9 cardinal positions as well as with cross-cover testing. Other tests include the Titmus housefly test, the Lang stereo test, the Hess screen test, red-filter test, Maddox rod evaluation, and Lancaster red-green test. ${ }^{12,30}$ In young children, who may be less cooperative with an examiner, binocular gaze conjugacy may only be assessable with simpler algorithms, such as following an object moving in a set trajectory. ${ }^{9}$ When such tests are performed in conjunction with the remainder of the neuro-ophthalmic and physical evaluation, one can localize neurological lesions and quantitate ocular motility deficits, particularly those associated with TBI, ${ }^{18}$ with great accuracy. Despite this capability, other than assessment of gaze in the cardinal positions, these tests are not used routinely in the emergency setting due to the need for a trained practitioner to administer them, the requirement for sophisticated equipment, and the urgent nature of many neurological disorders.

Unlike these techniques, our technology does not examine the full range of ocular motility. If the subject's eyes are positioned $55 \mathrm{~cm}$ from the center of the $30 \times 35-\mathrm{cm}$ viewing monitor, this algorithm elicits pupil movement in a maximum range of approximately $15^{\circ}$ in any direction from midposition, or approximately $30^{\circ}$ total from top to bottom or side to side. Thus, the algorithm does not require or assess the full range of ocular motility, nor the entire visual field. Use of a larger monitor, or one positioned closer to the subject, would enable assessment of these.

A second limitation is that our technique has not been rigorously compared with gold-standard physical examination or scleral coil techniques. Another potential limitation of this study is lack of formal visual acuity testing in controls as well as test subjects. Because subjects removed glasses and toric lenses for viewing to optimize the ability of the eye-tracking camera to find the pupil, some may have experienced impaired vision. Future studies will be needed to clarify the acuity necessary for optimal tracking. Patients with mass lesions were tracked under the same conditions (no glasses) each time they underwent tracking, so it is unlikely that visual acuity accounts for their alterations in eye tracking.

One might wonder if the eye-tracking deficits we are seeing in patients with mass lesions are due to inattention, psychomotor disturbance, or other spurious events. Arguing strongly against this is the fact that abnormalities were demonstrated not in 1 cycle, as the eyes followed the video aperture circumnavigating the monitor, but in 5 serial cycles. If a person were inattentive, the probability that they would be inattentive in exactly the same pattern for 5 consecutive times is low. This is further illustrated in Fig. 4B, in which the same patient's data are tracked over 35 days and plotted as a cartesian coordinate on the $y$ axis versus time on the $\mathrm{x}$ axis. Not only are the eyes moving in similar patterns over cycles within the same tracking session, but also between tracking sessions as the tracking disorder steadily improves over 35 days. In all cycles the disruption is in the same regions. Such a finding would be consistent with an intrinsic pathological entity causing the eye-tracking problem, as opposed to a volitional act by the subject being tracked.

Our methodology assesses physiological functioning of the CNS, and thus is different from imaging technologies enabling visualization of brain anatomy, or monitors that assess pressure rather than the impact of elevated pressure. Our methodology is also different from technologies such as the electroencephalogram that measures electrical activity. Eye tracking most closely resembles physical examination of a subject, which also yields information regarding physiological functioning. Unlike a physical examination, however, eye tracking does not necessarily require a trained examiner and could be performed remotely or in an automated fashion. Conceivably one could assess a patient with hydrocephalus for a shunt malfunction by having them watch a video on their home computer.

Unlike conventional eye tracking, in which subjects follow instructions to be deliberately calibrated and thus are conscious that their eye movements are being tracked, nonspatially calibrated tracking could occur via a webcam while someone is reading or watching a video. It does not require that the subject explicitly consent to being tracked prior to assessment of their CNS functioning, and thus raises ethical considerations. Dementia, Parkinson disease, progressive supranuclear palsy, schizophrenia, amyotrophic lateral sclerosis, autism, and fragile X syndrome 
are among the numerous diseases with characteristic anomalies that are detectable using eye-movement tracking technology. $4,20,21,23,24,27,32$

\section{Conclusions}

In this paper we present proof of concept data supporting a method for tracking eye movements while a subject watches television or a video that reveals weakness of the abducent and oculomotor nerves. This represents the first report in the literature of the use of eye tracking to assess physiological functioning of the CNS. This method may potentially serve as a proxy for physical examination, could potentially be performed remotely in the absence of a trained examiner, and may have utility for evaluation of diseases that impact $\mathrm{CN}$ function, such as hydrocephalus and brain injury.

\section{Acknowledgments}

Dr. Samadani's work is supported in part by the Department of Veterans Affairs (VA), Veterans Health Administration, Office of Research and Development, Rehabilitation Research and Development Service, via a VA Merit Award (1I01RX000319-01), and she was supported by a Career Development Award from the American College of Surgeons/Neurosurgery Research Education Foundation of the AANS. She is also supported by the Steven and Alexandra Cohen Center for Veteran Post-Traumatic Stress and Traumatic Brain Injury; by the Thrasher Research Fund; and by Research to Prevent Blindness.

\section{References}

1. Adler DE, Milhorat TH: The tentorial notch: anatomical variation, morphometric analysis, and classification in 100 human autopsy cases. J Neurosurg 96:1103-1112, 2002

2. Alemdar M, Kamaci S, Budak F: Unilateral midbrain infarction causing upward and downward gaze palsy. J Neuroophthalmol 26:173-176, 2006

3. Altintas O, Etus V, Etus H, Ceylan S, Caglar Y: Risk of strabismus and ambylopia in children with hydrocephalus. Graefes Arch Clin Exp Ophthalmol 243:1213-1217, 2005

4. Archibald NK, Hutton SB, Clarke MP, Mosimann UP, Burn DJ: Visual exploration in Parkinson's disease and Parkinson's disease dementia. Brain 136:739-750, 2013

5. Beaufils E, Corcia P, de Toffol B, Praline J: Occurrence of eye movement disorders in motor neuron disease. Amyotroph Lateral Scler 13:84-86, 2012

6. Brodsky MC: Surgical treatment of vertical ocular motility disorders of supranuclear origin. Am Orthopt J 61:28-33, 2011

7. Brodsky MC: Three dimensions of skew deviation. Br J Ophthalmol 87:1440-1441, 2003

8. Caeyenberghs K, Leemans A, Geurts M, Taymans T, Vander Linden C, Smits-Engelsman BC, et al: Brain-behavior relationships in young traumatic brain injury patients: fractional anisotropy measures are highly correlated with dynamic visuomotor tracking performance. Neuropsychologia 48:14721482,2010

9. Cavézian C, Vilayphonh M, de Agostini M, Vasseur V, Watier L, Kazandjian S, et al: Assessment of visuo-attentional abilities in young children with or without visual disorder: toward a systematic screening in the general population. Res Dev Disabil 31:1102-1108, 2010

10. Ciuffreda KJ, Han Y, Kapoor N, Ficarra AP: Oculomotor rehabilitation for reading in acquired brain injury. NeuroRehabilitation 21:9-21, 2006
11. Clark D, Eggenberger E: Neuro-ophthalmology of movement disorders. Curr Opin Ophthalmol 23:491-496, 2012

12. da Cunha Matta AP, Nunes G, Rossi L, Lawisch V, Dellatolas G, Braga L: Outpatient evaluation of vision and ocular motricity in 123 children with cerebral palsy. Dev Neurorehabil 11:159-165, 2008

13. Dagi LR, Zoumalan CI, Konrad H, Trokel SL, Kazim M: Correlation between extraocular muscle size and motility restriction in thyroid eye disease. Ophthal Plast Reconstr Surg 27:102-110, 2011

14. Deleu D, Buisseret T, Ebinger G: Vertical one-and-a-half syndrome. Supranuclear downgaze paralysis with monocular elevation palsy. Arch Neurol 46:1361-1363, 1989

15. Dennis M, Fitz CR, Netley CT, Sugar J, Harwood-Nash DC, Hendrick EB, et al: The intelligence of hydrocephalic children. Arch Neurol 38:607-615, 1981

16. Friedman DI: Pearls: diplopia. Semin Neurol 30:54-65, 2010

17. Friedman DI, Jankovic J, McCrary JA III: Neuro-ophthalmic findings in progressive supranuclear palsy. J Clin Neuroophthalmol 12:104-109, 1992

18. Goodrich GL, Flyg HM, Kirby JE, Chang CY, Martinsen GL: Mechanisms of TBI and visual consequences in military and veteran populations. Optom Vis Sci 90:105-112, 2013

19. Hanson RA, Ghosh S, Gonzalez-Gomez I, Levy ML, Gilles FH: Abducens length and vulnerability? Neurology 62:3336,2004

20. Hicks SL, Robert MP, Golding CV, Tabrizi SJ, Kennard C: Oculomotor deficits indicate the progression of Huntington's disease. Prog Brain Res 171:555-558, 2008

21. Kennedy DP, Adolphs R: Impaired fixation to eyes following amygdala damage arises from abnormal bottom-up attention. Neuropsychologia 48:3392-3398, 2010

22. Kerrison JB, Maumenee IH: Neuro-ophthalmic genetics. Curr Opin Ophthalmol 8:35-40, 1997

23. Levy DL, Sereno AB, Gooding DC, O'Driscoll GA: Eye tracking dysfunction in schizophrenia: characterization and pathophysiology. Curr Top Behav Neurosci 4:311-347, 2010

24. Marx S, Respondek G, Stamelou M, Dowiasch S, Stoll J, Bremmer F, et al: Validation of mobile eye-tracking as novel and efficient means for differentiating progressive supranuclear palsy from Parkinson's disease. Front Behav Neurosci 6:88, 2012

25. Mikheev A, Nevsky G, Govindan S, Grossman R, Rusinek H: Fully automatic segmentation of the brain from T1-weighted MRI using Bridge Burner algorithm. J Magn Reson Imaging 27:1235-1241, 2008

26. Nathan H, Ouaknine G, Kosary IZ: The abducens nerve. Anatomical variations in its course. J Neurosurg 41:561-566, 1974

27. Pelak VS: Ocular motility of aging and dementia. Curr Neurol Neurosci Rep 10:440-447, 2010

28. Pierrot-Deseilligny C: Nuclear, internuclear, and supranuclear ocular motor disorders. Handb Clin Neurol 102:319-331, 2011

29. Ritter AM, Muizelaar JP, Barnes T, Choi S, Fatouros P, Ward J, et al: Brain stem blood flow, pupillary response, and outcome in patients with severe head injuries. Neurosurgery 44:941-948, 1999

30. Rogers GL, Jordan CO: Pediatric vision screening. Pediatr Rev 34:126-132, 2013

31. Rudolph G, Nentwich M, Hellebrand H, Pollack K, Gordes R, Bau V, et al: KIF21A variant R954W in familial or sporadic cases of CFEOM1. Eur J Ophthalmol 19:667-674, 2009

32. Sharma R, Hicks S, Berna CM, Kennard C, Talbot K, Turner MR: Oculomotor dysfunction in amyotrophic lateral sclerosis: a comprehensive review. Arch Neurol 68:857-861, 2011

33. Suh M, Basu S, Kolster R, Sarkar R, McCandliss B, Ghajar $\mathrm{J}$ : Increased oculomotor deficits during target blanking as 
an indicator of mild traumatic brain injury. Neurosci Lett 410:203-207, 2006

34. Tamhankar MA, Kim JH, Ying GS, Volpe NJ: Adult hypertropia: a guide to diagnostic evaluation based on review of 300 patients. Eye (Lond) 25:91-96, 2011

35. Vaphiades MS, Bhatti MT, Lesser RL: Ocular myasthenia gravis. Curr Opin Ophthalmol 23:537-542, 2012

36. Yang AI, Balser DS, Mikheev A, Offen S, Huang JH, Babb $\mathrm{J}$, et al: Cerebral atrophy is associated with development of chronic subdural haematoma. Brain Inj 26:1731-1736, 2012

37. Zeiner HK, Prigatano GP, Pollay M, Biscoe CB, Smith RV: Ocular motility, visual acuity and dysfunction of neuropsychological impairment in children with shunted uncomplicated hydrocephalus. Childs Nerv Syst 1:115-122, 1985

\section{Author Contributions}

Conception and design: Samadani, Farooq, Ritlop, Huang. Acquisition of data: Samadani, Farooq, Warren, Reyes, Lamm, Alex, Nehrbass, Kolecki, Jureller, Schneider, Chen, Shi, Mendhiratta, Kwak, Mikheev, Rusinek, George, Smith. Analysis and interpretation of data: Samadani, Farooq, Alex, Jureller, Shi,
Mendhiratta, Mikheev, Rusinek, George, Kondziolka. Drafting the article: Samadani, Alex, Nehrbass, Schneider, Chen, Shi. Critically revising the article: Samadani, Warren, Schneider, Chen, Kwak, Kondziolka, Huang. Reviewed submitted version of manuscript: Samadani, Farooq, Ritlop, Warren, Reyes, Lamm, Alex, Nehrbass, Kolecki, Schneider, Chen, Shi, Mendhiratta, Huang, Qian, Kwak, Rusinek, George, Fergus, Kondziolka, Huang, Smith. Approved the final version of the manuscript on behalf of all authors: Samadani. Statistical analysis: Lamm, Jureller, Shi, Qian, Fergus, Huang. Administrative/technical/ material support: Samadani, Ritlop, Reyes, Nehrbass, Kolecki. Study supervision: Samadani, Warren, Fergus, Smith.

\section{Supplemental Information \\ Previous Presentation}

Portions of this work were presented in abstract form (poster) at the Association for Research in Vision and Ophthalmology, May 5, 2014, in Orlando, Florida.

\section{Correspondence}

Uzma Samadani, 423 E. 23rd St., MC 112, New York, NY 10010.email: uzma.samadani@nyumc.org. 